\title{
A Wear Prediction Model for Spur Gears Based on the Dynamic Meshing Force and Tooth Profile Reconstruction
}

\author{
Zhouli Zhang ${ }^{1, a}$, Changsong Zheng ${ }^{1,2, b}$, Mengquan Wen ${ }^{3, c}$, Shuo Yang ${ }^{3, d}$, \\ Huizhu $\mathrm{Li}^{1, \mathrm{e}}$ and Qiu Du ${ }^{1, \mathrm{f}}$
}

\author{
${ }^{1}$ School of Mechanical Engineering, Beijing Institute of Technology, Beijing 100081, China \\ ${ }^{2}$ Collaborative Innovation Center of Electric Vehicle in Beijing, Beijing 100081, China \\ 3 Jianglu Machinery \& Electronics Group Corporation, Hunan 411100, China \\ azhang_zl93@163.com, bzhengyuanye@126.com, cwmq_83@163.com, djjyang@qq.com, \\ elhz20041471@163.com, fduqiu@bit.edu.cn
}

Keywords: Dynamic meshing force, Reconstruction of tooth profile, Wear, Archard's wear equation.

\begin{abstract}
In this study, Archard's wear equation is combined with a nonlinear dynamic model and a reconstraction method for the wear tooth profile to predict the wear depth of gears. The dynamic model, which is used to determine the dynamic meshing force, and the reconstraction of the wear tooth profile serves as the basis of the sliding coefficient calculation. Next, the dynamic meshing force and sliding coefficient are used to calculate the surface wear in Archard's wear equation. Then, the dynamic meshing force and sliding coefficient would be recalculated according to the surface wear state. After multiple iterations of previous steps, the simulation results show that the non-uniform wear of the gear surface has a great influence on the distribution of dynamic meshing force, and will increase significantly the peak of dynamic meshing force. And in return, the changing dynamic meshing force would enhance the non-uniformity of wear. These two factors influence and exaggerate each other, but are limited by sliding coefficient. When the driving gear runs after 858 million cycles accumulatively, the maximum wear depth reaches $0.0737 \mathrm{~mm}$, and the peak value of dynamic meshing force is more than 4 times of the unweared, which exists the risk of overload. The proposed model can be used to predict gears wear life and design gears, which has a certain engineering significance.
\end{abstract}

\section{Introduction}

Gear system is widely uesd in various mechanical transmission devices. But wear, which is one of the most important factors that affect the behavior of gear systems, would cause gear systems to fail. The traditional experimental method to study the wear life of gears, whose results lack universality, demands a mountain of expenses and time ${ }^{[1]}$. Therefore, applying the numerical simulation technology to the study of wear is an effective alternative ${ }^{[2]}$.

Flodin established a simulation model for mild wear of gears ${ }^{[3-4]}$. Bajpai improved the model proposed by Flodin, taking intentional surface modifications and manufacturing related imperfections into account ${ }^{[5]}$. Ding studied the two-way relationship between surface wear and gear dynamics ${ }^{[6]}$. He Rongguo considered the effect of temperature on wear, and gave the calculation formula of the material wear rate ${ }^{[7]}$. Zhang Yicheng developed a wear calculation formula based on friction wear work principles, which only need to calculate the macro force rather than instaneous contact pressure $^{[8]}$. In addition, there are also several other wear models based on deffierent theories ${ }^{[1,9-10]}$. At present, these studies are mainly using meshing force or stress which is calculated under quasi-static conditions as the basis for the calculation of wear. But in fact, the gear systems usually show complex nonlinear dynamic characteristics ${ }^{[11]}$. The actual meshing force, that is, the dynamic meshing force, varies significantly with the gear speed and surface wear, while the static meshing force does not vary with the speed change and isn't sensitive to surface wear. So the wear models under quasi-static conditions are not very accurate. 
Therefore, a wear prediction model for involute spur gears is proposed in this paper, which combines Archard's wear equation, a gear nonlinear dynamic model and a reconstraction method for the wear tooth profile. The dynamic model is used to calculate the dynamic meshing force, while the sliding coefficient is determined by involute profile geometry. As the result of the fact that the gear surface wear would significantly change the dynamic meshing force and sliding coefficient, the calculated wear amount would be regarded as the tooth profile errors, which would be considered in the gear nonlinear dynamic model to recalculate the dynamic meshing force. And the tooth profile would also be reconstructed according to the surface wear in order to get the new sliding coefficient. Repeat iterations, then the simulation results would be used to predict the wear depth and study the variation trend of meshing force.

\section{Gear Wear Model}

When the study object is a pair of gears, the mechanism of wear is shown in Fig. 1. Assume the face width of driving and driven gears are $B$. During time $\mathrm{d} t$, the contact point on the driving gear moves from point $P_{j}$ to point $P_{j+1}$, with a moving distance $\mathrm{d} s^{p}{ }_{j}$, which results in a normal wear depth $\mathrm{d} h^{p}$ and a wear volume $\mathrm{d} V_{j}{ }_{j}$. At the same time, the contact point on the driven gear moves from point $G_{j}$ to point $G_{j+1}$, with a moving distance $\mathrm{d} s^{g}$, which leads to a wear depth $\mathrm{d} h^{g_{j}}$ and a wear volume $\mathrm{d} V^{g}$ on the driven gear. According to the geometey, the wear volume of driving gear $\mathrm{d} V^{p}$ and the sliding distance $\mathrm{d} s$ can be written as

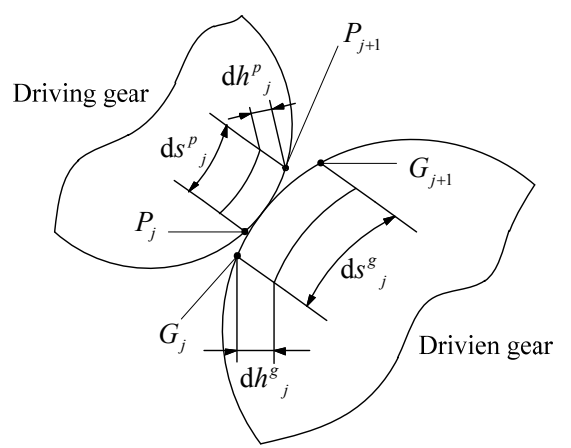

Fig. 1 Gear wear schematic diagram

$\mathrm{d} V^{p}{ }_{j}=B \cdot \mathrm{d} h^{p}{ }_{j} \cdot \mathrm{d} s^{p}{ }_{j}, \mathrm{~d} s=\left|\mathrm{d} s^{p}{ }_{j}-\mathrm{d} s^{g}{ }_{j}\right|$

Put the $\mathrm{d} V^{p}$ and $\mathrm{d} s$ into the Archard's wear equation $\left(\frac{\mathrm{d} V}{\mathrm{~d} s}=K \frac{F}{H}\right)$, then the wear depth of driving gear can be express as

$$
\mathrm{d} h^{p}{ }_{j}=\frac{K \cdot F}{B \cdot H} \cdot \frac{\left|\mathrm{d} s_{j}{ }_{j}-\mathrm{d} s^{g}{ }_{j}\right|}{\mathrm{d} s^{p}{ }_{j}}=\frac{K \cdot F}{B \cdot H} \cdot \varepsilon^{p}{ }_{j}
$$

where $K$ is the dimensionless wear coefficient; $F$ is the normal force; $H$ is the tooth surface hardness; $\varepsilon_{j}$ is the sliding coefficient of driving gear at the piont $P_{j}$. Similarly, the wear depth of driven gear at the point $G_{j}$ can also get.

Since the numerical simulation can be only performed at a large number of discrete positions, the tooth profile participating in the meshing process would be discretized to 300 points accordingly. More details could refer to Ref. [8]. Besides, the simulation time would be discretized to a series of time steps, as the system is time-varying. The threshold of the wear depth is set as $1 \mu \mathrm{m}^{[10]}$. Retain the parameters of each pair of meshing points within a time step until the wear of any points on the gears reaches the threshold. Then continue the next time step.

At any point $j$ after $n$ time steps, the total wear depth of the driving gear $h^{p}$ and driven gear $h^{g}$ can be expressed as 


$$
\begin{gathered}
h_{j}^{p}=\sum_{n=1}^{N} h_{j, n}^{p}=\frac{K}{B \cdot H} \sum_{n=1}^{N} F_{j, n} \cdot \varepsilon_{j, n}^{p} \cdot n^{p} \cdot \Delta t_{n} \\
h_{j}^{g}=\sum_{n=1}^{N} h_{j, n}^{g}=\frac{K}{B \cdot H} \sum_{n=1}^{N} F_{j, n} \cdot \varepsilon_{j, n}^{g} \cdot n^{g} \cdot \Delta t_{n}
\end{gathered}
$$

where $F_{j, n}$ is the normal force; $\varepsilon_{j, n}$ and $\varepsilon^{g_{j, n}}$ are the sliding coefficients; $n^{p}$ and $n^{g}$ are the average rotate speeds; $\Delta t_{n}$ is the duration of the $n$-th time step. Here, the superscript $p$ represents the driving gear (gear $p$ ), and $g$ represents the driven gear (gear $g$ ). The subscript $j$ represents the $j$-th discrete point, and $n$ represents the $n$-th time step.

\section{The Dynamic Model of Gear System}

The nonlinear dynamic model of gear system is shown in Fig. 2, which consists of two rigid wheels.

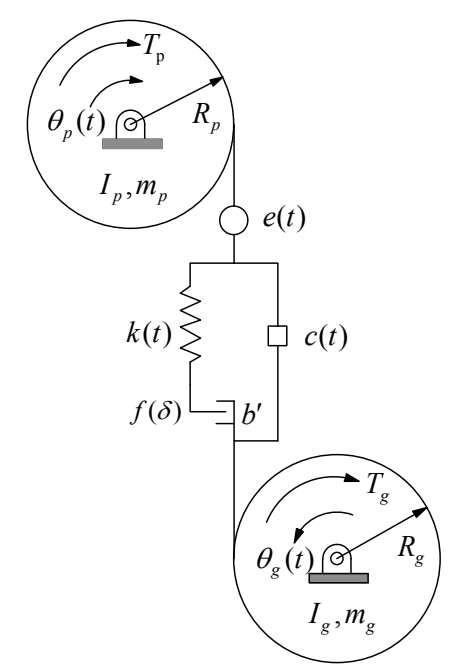

Fig. 2 Dynamic model of gear system

Where $I_{p}$ and $I_{g}$, are inertias; $m_{p}$ and $m_{g}$ are masses; $R_{p}$ and $R_{g}$ are base circle radius. Each wheel is supported by a rigid shaft. As a result, the system only has two degrees of freedom, i.e. angular displacement of gear $p \theta_{p}(t)$ and angular displacement of gear $g \theta_{g}(t)$. And a periodically time-varying mesh stiffness $k(t)$ and a viscous damping $c(t)$ connect the two gear wheels along the line of action. A blacklash function $f(\delta)$ is included to represent the amount of clearance between mated gear teeth, and an internal displacement excitation $e(t)$ is also applied at the gear mesh interface to represent manufacturing errors, intentional modifications of tooth profile or a wear profile. The backlash function in the nonlinear dynamic system can be written as ${ }^{[12]}$

$$
f(\delta)=\left\{\begin{array}{lc}
\delta & (\delta \geq 0) \\
0 & \left(-b^{\prime}<\delta<0\right) \\
\delta+b^{\prime} & \left(\delta \leq-b^{\prime}\right)
\end{array}\right.
$$

where $\delta\left(\delta=R_{p} \theta_{p}(t)-R_{g} \theta_{g}(t)-e(t)\right)$ is the dynamic transmission error. The gear backlash $b^{\prime}$ can be expressed as $b^{\prime}=2 a_{0} \cos \alpha \cdot\left(i n v \alpha^{\prime}-i n v \alpha\right)+b_{0}$, in which $a_{0}$ is the ideal operating center distance; $\alpha$ is the pressure angle; $\alpha^{\prime}$ is the wroking pressure angle of pitch circle; $b_{0}$ is the gear backlash due to the change of actual tooth thickness on the pitch circle, $b_{0}=0$ is considered in this paper.

In consideration of the fact that the amount of meshing tooth pairs at the same time maybe more than one, a subscript $i$ is introduced to represent the $i$-th meshing tooth pair. And as for the periodically time-varying mesh stiffness of single meshing teeth pair, i.e. $k_{i}(t)$, material mechanics method would be uesd here to calculate $k_{i}(t)$. According to Cornell ${ }^{[13]}$, the total compliance or flexibility of a gear tooth at the point of load is made up of three deflections: 1) the basic tooth as a cantilever beam, $\delta_{B j}{ }^{p}$ and $\delta_{B j}{ }^{g} ; 2$ ) the fillet and foundation, $\delta_{M j}{ }^{p}$ and $\delta_{M j}{ }^{g}$; and 3) the local contact and 
compression, $\delta_{h j}$. Each deflection for any meshing tooth pair $i$ can be calculated by the following equations:

$$
\begin{aligned}
& \delta_{B j}^{p, g}=\frac{F}{E \cdot B} \cos ^{2} \beta_{j}\left[10.92 \sum_{k=1}^{n} T_{k} \frac{L_{k j}{ }^{2}}{\left(2 y_{k}\right)^{3}}+3.1\left(1+0.294 \tan ^{2} \beta_{j}\right) \sum_{k=1}^{n} \frac{T_{k}}{2 y_{k}}\right] \\
& \delta_{M j}^{p, g}=\frac{F \cdot \cos ^{2} \beta_{j}}{B \cdot E}\left[\frac{16.67}{\pi}\left(\frac{L_{f}}{H_{f}}\right)^{2}+2(1-\lambda)\left(\frac{L_{f}}{H_{f}}\right)+1.534\left(1+\frac{\tan ^{2} \beta_{j}}{2.4(1+\lambda)}\right)\right] \\
& \delta_{h j}=\frac{4\left(1-\lambda^{2}\right)}{\pi E} \frac{F}{B}\left\{\left[\operatorname{In}\left(2 \frac{\sqrt{h_{1} h_{2}}}{b}\right)-\frac{\lambda}{2(1-\lambda)}\right]+\frac{\left(1-\lambda_{2}{ }^{2}\right)}{E_{2}}\right\}
\end{aligned}
$$

Then the periodically time-varying mesh stiffness at the $j$-th $(j=1,2,3, \ldots .300)$ point can be calculated by

$$
k_{j}=\frac{F}{{\delta_{M j}}^{p}+\delta_{M j}{ }^{g}+\delta_{B j}{ }^{p}+\delta_{B j}{ }^{g}+\delta_{h j}}
$$

More details can refer to Refs. [13-14]. And the calculation of $k_{i}(t)$ can be accomplished by calculating the stiffness of all the contact points and combining them together. Afterwards, the viscous damping $c_{i}(t)$ can be also determined by ${ }^{[12]}$

$$
c_{i}(t)=2 \xi \sqrt{k_{i}(t) \frac{m_{p} m_{g}}{m_{p}+m_{g}}}
$$

where $\xi$ is the damping ratio, $\xi=0.06$ is used in this paper empirically.

Accordingly, the dynamic meshing force $F_{i}$ then can be expressed as

$$
F_{i}=k_{i}(t) \cdot f\left(R_{p} \theta_{p}(t)-R_{g} \theta_{g}(t)-e(t)\right)+c_{i}(t) \cdot f\left(R_{p} \dot{\theta}_{p}(t)-R_{g} \dot{\theta}_{g}(t)-\dot{e}(t)\right)
$$

Assuming the input torque $T_{p}$ and output torque $T_{g}$ are constant and taking the friction torques into account, the equations of the nonlinear model are

$$
\left\{\begin{array}{l}
I_{p} \ddot{\theta}_{p}=T_{p}-R_{p} \sum_{i=1}^{n_{z}} F_{i}-\sum_{i=1}^{n_{z}} \Lambda_{i} \rho_{p i} \mu_{i} F_{i} \\
I_{g} \ddot{\theta}_{g}=R_{g} \sum_{i=1}^{n_{z}} F_{i}+\sum_{i=1}^{n_{z}} \Lambda_{i} \rho_{g i} \mu_{i} F_{i}-T_{g}
\end{array}\right.
$$

where $n_{z}$ is the amount of pairs of the teeth engaged at the same time, $n_{z}=2$ is considered in this paper; $\mu_{i}$ is the friction coeffcient; $\Lambda_{i}$ is the direction coefficient of the friction torque which is determined by the nominal sliding velocity. $\rho_{p i}$ and $\rho_{g i}$ are friction arms of the meshing tooth pair $i$, and can be defined as

$$
\begin{aligned}
& \rho_{p i}=a^{\prime} \sin \alpha^{\prime}-\sqrt{R_{a g}{ }^{2}-R_{g}{ }^{2}}+R_{p} w_{p} t \\
& \rho_{g i}=\sqrt{{R_{a g}{ }^{2}-R_{g}{ }^{2}}^{2}-R_{p} w_{p} t}
\end{aligned}
$$

where $R_{a g}$ is the addendum circle radius of gear $g ; w_{p}$ is the average rotate speed.

In addition, during gear meshing, even in a stable work condition, the actual rotate speeds of the gear pair, $\dot{\theta}_{\mathrm{p}}$ and $\dot{\theta}_{\mathrm{g}}$ will fluctuate, which generates inertia torques. If the rotate speeds of the gear pair remained canstant during the meshing process, Eq. (19) would become a static equation, which can be used to solve the meshing force under quasi-static conditions.

\section{The Calculation of Sliding Coefficients after Wear}

\subsection{The Reconstruction Process of the Wear Tooth Profile}

To discreble the tooth changing profile and the whole meshing process, two moving coordinate systems, $x_{p} O_{p} y_{p}$ and $x_{g} O_{g} y_{g}$, and one fixed coordinate system, $x O_{p} y$, are established, as shown in Fig. 3. The coordinate system $x_{p} O_{p} y_{p}$ is attached to gear $p$, and the axis $y_{p}$ is in the direction of $O_{p} M_{1}$. 
Similarly, the coordinate system $x_{g} O_{g} y_{g}$ is attached to gear $g$, and the axis $y_{g}$ is in the direction of $\mathrm{O}_{g} \mathrm{M}_{2}$. The two moving coordinate systems connect the fixed coordinate system by two time-varying angles, $\phi^{p}$ and $\phi^{g}{ }_{j}$ respectively. $\phi^{p}{ }_{j}$ and $\phi^{g}{ }_{j}$ can be expressed as ${ }^{[8]}$

$$
\phi_{j}^{p}=-\alpha^{\prime}+\tan \alpha_{j}^{p}, \quad \phi_{j}^{g}=-\alpha^{\prime}-\pi+\tan \alpha_{j}^{g}
$$

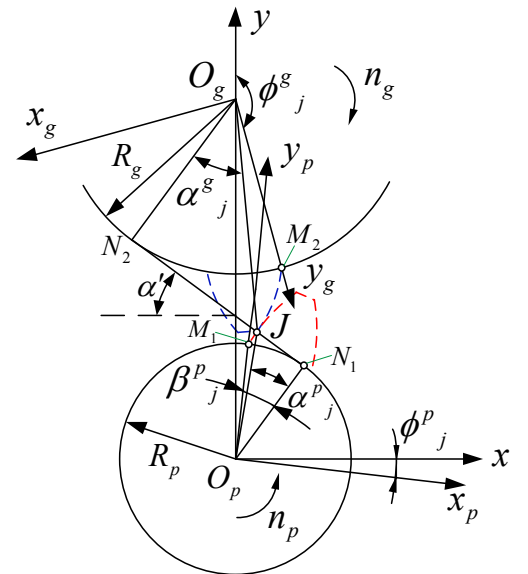

Fig. 3 Coordinate systems diagram

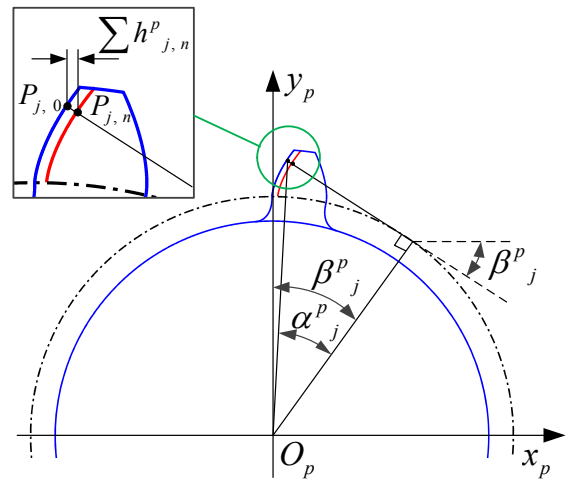

Fig. 4 Reconstruction of tooth profile

where $\alpha^{p_{j}}\left(\alpha^{g} j\right)$ is the pressure angle of the gear $p$ (gear $g$ ) at the $j$-th point.

Take gear $p$ as an example to show the method of the reconstruction process of the wear tooth profile. As shown in Fig. 4, the coordinate of the point $P_{j, n}$ in $x_{p} O_{p} y_{p}$, whose displacement used to be $P_{j, 0}$ before wear, can be expressed as

$$
\left\{\begin{array}{l}
{ }^{(1)} x_{j, n}^{p}={ }^{(1)} x_{j, 0}^{p}-\left(\sum h_{j, n}^{p}\right) \cdot \cos \beta_{j}^{p} \\
{ }^{(1)} y_{j, n}^{p}={ }^{(1)} y_{j, 0}^{p}-\left(\sum h_{j, n}^{p}\right) \cdot \sin \beta_{j}^{p}
\end{array}\right.
$$

where $\left({ }^{(1)} x^{p_{j, n}},{ }^{(1)} y_{j, n}\right)$ is the coordinate of the point $P_{j, 0}$, the $j$-th point on gear $p$ before wear point, as the superscript (1) represents the coordinate system $x_{p} O_{p} y_{p}$, and the superscript (0) and (2) represent the coordinate system $x O_{p} y$ and $x_{g} O_{g} y_{g}$ respectively. $\Sigma h^{p}{ }_{j, n}$ is the the cumulative wear depth at the $j$-th point. $\beta_{j}^{p}\left(\beta_{j}^{p}=\tan \alpha^{p}\right)$ is the angle between the axis $y_{p}$ and line $O_{p} N_{1}$. The reconstruction would be done after calculating the coordinates of all the discrete points.

\subsection{Sliding Coefficient}

The sliding coefficient is used to indicate the extent of the relative slip between the teeth surface, which is related to the position of the gear. Along with the wear of the gear, the meshing position on the gears moves from initial pair of discrete points to a new pair. Therefore, the discrete points with wear on gear $p$ would be regarded as the benchmark, and the meshing points on gear $g$ would be redefined to determine the new sliding coefficient.

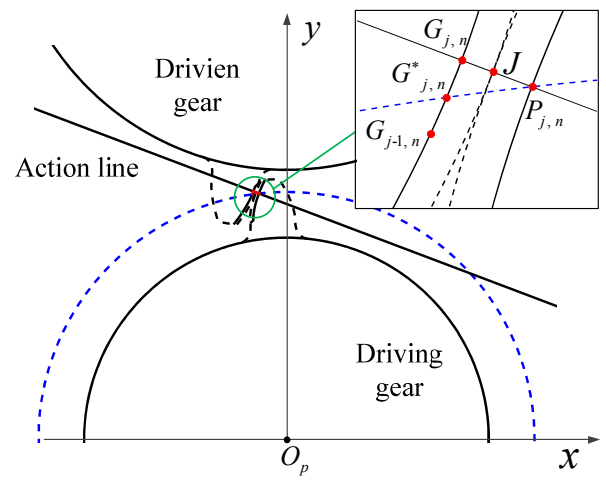

Fig. 5 Illustration of the reconstruction of contact points

As shown in Fig. 5, the black dotted lines represent the teeth profile without wear, and the $j$-th point on gear $p$ exactly contacts with the $j$-th point on gear $g$. But the two points would not contact anymore as the $j$-th point on gear $p$ moved to point $P_{j, n}$, and the $j$-th point on gear $g$ moved to point $G_{j, n}$. The black solid lines represent the teeth profile after wear. 
Assume point $P_{j, n}$ contacts with the point $G_{j, n}^{*}$, then the first step is to determine the approximate position of point $G_{j, n}^{*}$ geometrically (in Fig. $5, G_{j, n}^{*}$ is between points $G_{j, n}$ and $G_{j-1, n}$ ). Hence, the coordinate of $G_{j, n}^{*}$ in $x O_{p} y$, i.e. $\left({ }^{(0)} x_{j, n}^{*},{ }^{(0)} y_{j, n}^{*}\right)$, satisfies the following equations

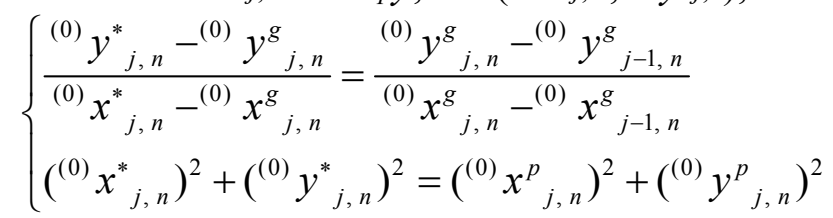

where $\left({ }^{(0)} x_{j, n},{ }^{(0)} y^{p_{j, n}}\right)$ is the coordinate of the point $P_{j, n}$ in $x O_{p} y ;\left({ }^{(0)} x^{g}{ }_{j, n},{ }^{(0)} y_{j, n}\right)$ is the coordinate of the point $G_{j, n}$ in $x O_{p} y ;\left({ }^{(0)} x^{g}{ }_{j-1, n},{ }^{(0)} y^{g}{ }_{j-1, n}\right)$ is the coordinate of the point $G_{j-1, n}$ in $x O_{p} y$. The coordinate of any point can be freely converted in the three coordinate systems ${ }^{[9]}$.

After determine all coordinates of the new contact points on gear $g$, convert the coordinates of all the points from $x O_{p} y$ to $x_{g} O_{g} y_{g}$. Then the distances of the two adjacent points can be calculated by

$$
\begin{aligned}
& \mathrm{d} s_{j, n}^{p}=\sqrt{\left({ }^{(1)} x_{j, n}^{p}-{ }^{(1)} x_{j-1, n}^{p}\right)^{2}+\left({ }^{(1)} y_{j, n}^{p}-{ }^{(1)} y_{j-1, n}^{p}\right)^{2}} \\
& \mathrm{~d} s_{j, n}^{g}=\sqrt{\left({ }^{(2)} x_{j, n}^{g}-{ }^{(2)} x_{j-1, n}^{g}\right)^{2}+\left({ }^{(2)} y_{j, n}^{g}-{ }^{(2)} y_{j-1, n}^{g}\right)^{2}}
\end{aligned}
$$

The new sliding coefficients after gears wear can be calculated by the define

$$
\varepsilon_{j, n}^{p}=\frac{\left|d s_{j, n}^{p}-d s_{j, n}^{g}\right|}{d s^{p}{ }_{j, n}}, \quad \varepsilon_{j, n}^{g}=\frac{\left|d s_{j, n}^{p}-d s^{g}{ }_{j, n}\right|}{d s^{g}{ }_{j, n}}
$$

It must be pointed out that the new sliding coefficients can be used in Archard's wear equation only after interference analysis. As shown in Fig. 6, keep gear $g$ from moving and let gear $p$ rotate an angle $\varphi_{j, n}$ to make the two points, $P_{j, n}$ and $G_{j, n}^{*}$, contact. If there are other points contacting, the sliding coefficients are not be right, and further analysis is required, otherwise the sliding coefficients could be considered right. Taking the deformation into account, an interference boundary would be established according to $f(\delta)$, which represents the amount of actual deformation between mated gear teeth at the contact points in essence. If the tooth profile of gear $p$ doesn't intersect the interference boundary, the values of sliding coefficients remain the same as the value calculated. Otherwise, as shown in Fig. 6, the values of sliding coefficients are set to zero.

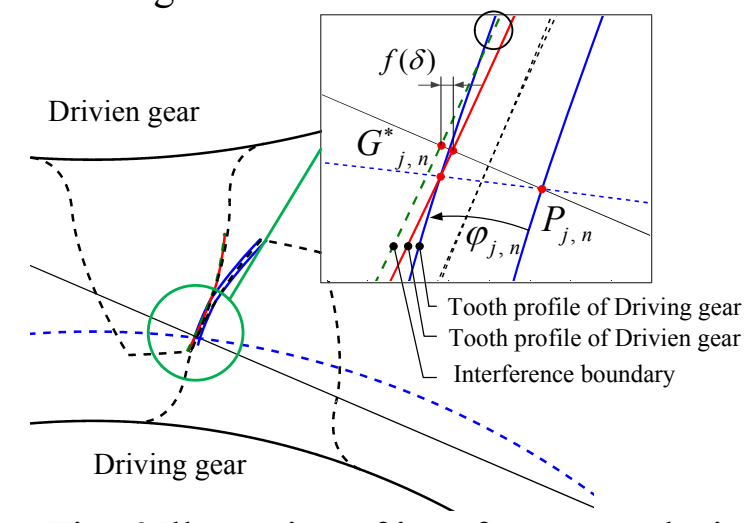

Fig. 6 Illustration of interference analysis

\section{Wear Simulation and Results Analysis}

The parameters of the gear system are shown in Table 1, and the flow chart of the computational methodology is shown in Fig. 7. The initial geometric description of the actual gear tooth surfaces serves as the initial state for the wear prediction. Here, $e(t)=0$ serves as the initial manufacturing errors. The next step is using 4-order Runge-Kutta method to solve the dynamic equation (11) to determine the dynamic meshing force. Then the sliding coefficients can be calculated by equations (17), and put the dynamic meshing force and sliding coefficients into Archard's wear equations (3) to get the wear depth. When the time step is over, given $e(t)=\Sigma\left(h_{\mathrm{j}, \mathrm{n}}^{\mathrm{p}_{\mathrm{j}}}+h_{\mathrm{j}, \mathrm{n}}^{\mathrm{g}}\right)$, reconstruct the actual teeth profiles and continue the next time step. With lots of iterative computations, several sets of simulation results are acquired to predict the wear depth and study the variation trend of meshing force. 
Table 1 Parameters of the gear system

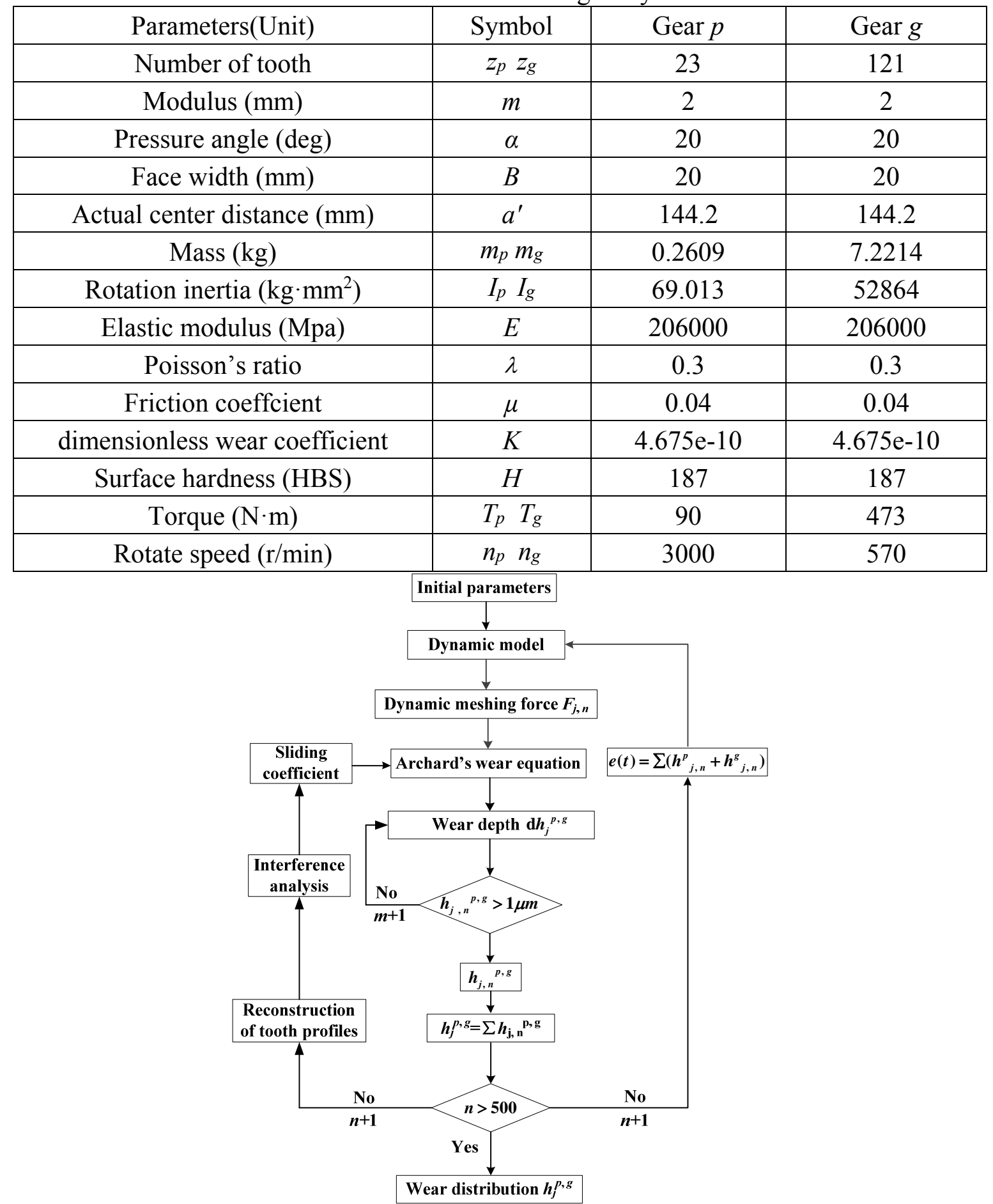

Fig. 7 Calculation flow chart

\subsection{The Effect of Rotate Speed on Meshing Force and Wear Depth}

As Fig. 8 shows, the abscissa represents the number of the 300 discrete points on driving gear; the dynamic meshing force is close to the static meshing force when the rotate speed of driving gear is low, except the significant fluctuation produced when there are tooth pairs coming into or exiting mesh. The trend of fluctuation becomes flatter as the rotate speed of driving gear becomes faster, and the distribution of dynamic meshing force begin to deviate from the static meshing force with the difference between the two becoming bigger and bigger. The reason for the difference between dynamic and static meshing forces is the inertia torques due to the instantaneous fluctuation of rotate speed, while the static meshing force is calculated regardless of inertia torques. 


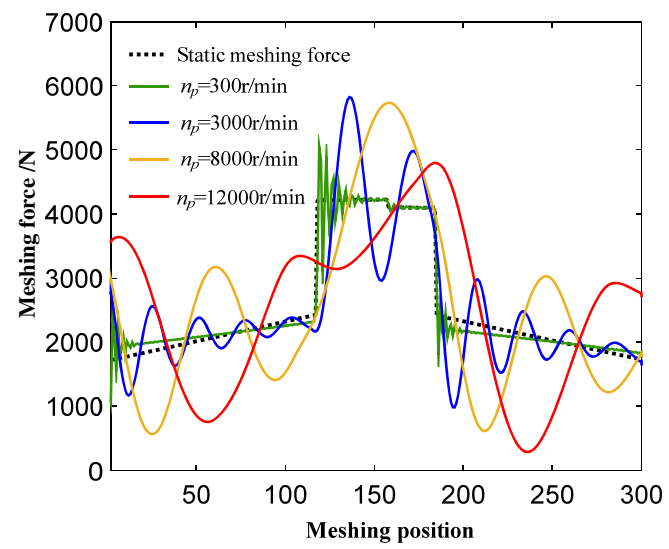

Fig. 8 Dynamic meshing force when driving gear runs at different speeds

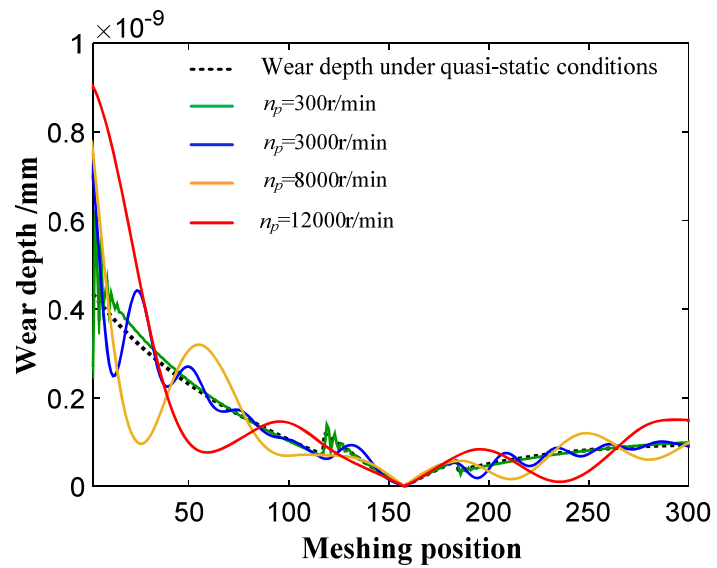

Fig. 9 Wear depth of driving gear meshing once at different speeds

Taking gear $p$ as an example to analyze the effect of rotate speed on wear, it is not difficult to discover that the force is one of the most important factors that affect wear depth according to Eq. (4). what's more, it is the only factor that changes with rotate speed, which leads to different dsitributions of wear depth. As Fig. 9 depicts, the effect of rotate speed on the wear depths is similar to that of the meshing force, which means, the wear depth, calculated basing on the model proposed in this paper, coincides with the distribution of wear depth under quasi-static conditions when the rotate speed is low, but the difference between them becomes larger as the rotate speed becomes faster. It is obvious that the wear model proposed in this paper can accurately reflect the influence of rotate speed on wear depth and the nonuniformity of wear distribution.

\subsection{The Variation Trend of Dynamic Meshing Force with Wear}

As shown in Fig. 10, besides slight changes in the peak and distribution, the dynamic meshing force doesn't change a lot at first. As the gears continue wearing, the peak and amplitude of the dynamic meshing force begin to increase significantly, and its distribution gradually becomes a series of convex peaks. The zero meshing force also appears, which indicates the separation phenomenon during the meshing process and worse work condition. In general, the distribution of dynamic meshing force changes significantly, and its peak and amplitude show great growth trends. However, as Fig. 11 shows, the trend of the peak is not monotonous, but increases with violent fluctuations and finally declines. In the later period, the peak of meshing force has reached 3 to 4 times as much as theoretical value $\left(=T_{p} / R_{p}\right)$, which leads to overload risk.


Fig. 10 Dynamic meshing forces of different time steps 




Fig. 11 The trend of the peak of meshing force

\subsection{The Trend of Wear Depth}

As Fig. 12(a) depicts, the successive order of wear severity on the driving gear is the root of the tooth, the top of the tooth, and the node, which is consistent with the fact. As the result of wear, there are several convex peaks and valleys forming on the tooth profile, which casues the value of $e(t)$ and its derivative fluctuating significantly, and eventually change the distribution of meshing force as mentioned earlier. Consequently, the changed dynamic meshing force would increase the non-uniformity of wear, they promote mutually. As the valleys on the tooth profile is likely not to contact with gear $g$ because of the interference due to the adjacent convex peaks, the sliding coefficients of the valleys become zero, which indicates these valleys would not wear in this time step. Therefore, the non-uniformity of wear could be alleviated, and the distribution of wear is the result of the balance of various factors. The distribution of wear depth of gear $g$ (Fig. 12(b)) is similar to that of driving gear, whereas the wear depth of gear $g$ is much smaller than that of gear $p$.



(a) driving gear

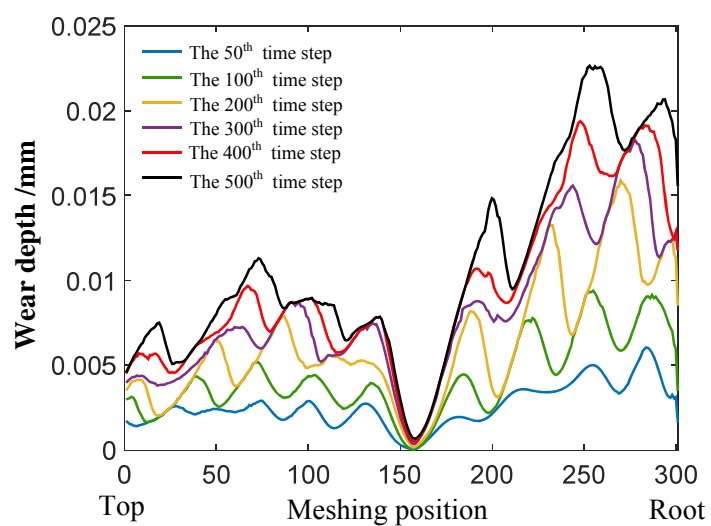

(b) driven gear

Fig. 12 Total wear depths of different time steps

\section{Conclusion}

In this study, a wear prediction model for involute spur gears is proposed, based on Archard's wear equation and a dynamic model of gear system. And a method to reconstruct the wear tooth profile and recalculate the sliding coefficient is presented here. Based on simulation results, the following conclusions can be made:

i) The dynamic meshing force varies greatly with the process of wear, as the peak and amplitude of the dynamic meshing force increase significantly. However, the trend of the peak is not monotonous and finally declines.

ii) As the result of wear, there are several convex peaks and valleys forming on the tooth profile, which causes the value of $e(t)$ and its derivative fluctuating significantly, and eventually change the distribution of meshing force as mentioned earlier. Consequently, the changed dynamic meshing force would increase the non-uniformity of wear, they promote mutually. However, the 
non-uniformity of wear could be alleviated, and the distribution of wear is the result of the balance of various factors.

iii) According to the whole process, when gear $p$ runs after 858 million cycles, the maximum wear depth reaches $0.0737 \mathrm{~mm}$, and the peak of dynamic meshing force is more than 4 times of the theoretical value, which leads to overload risk. At this time, the gear system can be considered failed due to wear, and the wear threshold can be determined accordingly, i.e. allowable limit wear depth. To be more specific, it can be concluded that the wear life of the gear system under this work condition is 858 million cycles.

\section{Acknowledgments}

This work was supported by the National Natural Science Foundation of China (Crant No. 51475044). And we would like to acknowledge Dr. Changsong Zheng, the author for correspondence, for his guidance during this research.

\section{References}

[1] PAN Dong, ZHAO Yang, LI Na, et al., The wear life prediction method of gear system, Journal of HarBin Institute of Technology. 44.9(2012)29-33.(in Chinese)

[2] LI Baoliang, Research of Numerical Simulation and Application of Line Contact Wear, Ph.D. thesis, Dalian Jiaotong University, 2010.(in Chinese)

[3] A. Flodin and S. Andersson, Simulation of mild wear in spur gears, Wear. 207.1-2(1997)16-23.

[4] A. Flodin and S. Andersson, A simplified model for wear prediction in helical gears, Wear. 249.3-4(2001)285-292.

[5] P.Bajpai, A. Kahraman, and N. E. Anderson, A Surface Wear Prediction Methodology for Parallel-Axis Gear Pairs, Journal of Tribology. 126.3(2004): 597-605.

[6] Ding $\mathrm{H}$ and $\mathrm{A}$. Kahraman, Interactions between nonlinear spur gear dynamics and surface wear, Journal of Sound \& Vibration. 307.3-5(2007): 662-679.

[7] HE Rongguo, JIANG Qinyu, YAO Yifu, Numerical Simulation of Tooth Wearing for Involute Helical Cylindrical Gears, Lubrication Engineering. 32.3(2007):88-91.(in Chinese)

[8] ZHANG Yicheng, Study on Tribology of Starved-Oil Gear Transmission, Ph.D. thesis, Central South University,2011.(in Chinese)

[9] JIN Feng, A Study on Involute Cylindrical Gear Wear Simulation System, Ph.D. thesis, Yanbian University,2013.(in Chinese)

[10] LIU Xianzeng, LU Qing, ZHANG Peng, et al., Modeling and Numerical Simulation of Surface Wear for Helical Gears, Journal of Anhui University of Technology(Natural Science). 32.2(2015)147-151.(in Chinese)

[11] GAO Hongbo, LI Yungong, LIU Jie, Dynamic analysis of a spur gear system with tooth-wear faults based on dynamic backlash, Journal of Vibration and Shock. 33.18(2014)221-226.(in Chinese)

[12] ZHANG Huibo, Research on Dynamics and Experiment of Gear Mechanism of Spacecraft with Multiple Clearances Coupled, Ph.D. thesis, Harbin Institute of Technology, 2015.(in Chinese)

[13] R. W. Cornell, Compliance and Stress Sensitivity of Spur Gear Teeth, Journal of Mechanical Design. 103.2(1983)447-459.

[14] P. Sainsot, P. Velex and O. Duverger, Contribution of Gear Body to Tooth Deflections-A New Bidimensional Analytical Formula, Journal of Mechanical Design. 126.4(2004)748-752. 